\title{
La Escuela de Enfermeras de Salus Infirmorum en el Hospital Español de Tánger
}

\author{
Concepción Mata Pérez \\ Profesora del CUE Salus Infirmorum de Cádiz (Adscrito UCA) \\ e-mail: conchi.mata@ca.uca.es
}

RESUMEN: La Escuela de Enfermeras de Salus Infirmorum en Tánger fue reconocida por el Ministerio de Asuntos Exteriores para la formación de mujeres en el campo de la enfermería durante el protectorado español. El objetivo que nos marcamos es poner de manifiesto la superación de la formación reglada de estas alumnas. Nuestra metodología se ha basado fundamentalmente en la consulta de los archivos propios de Salus Infirmorum, que nos han llevado a los fondos del Archivo de la Universidad de Cádiz, enriqueciendo nuestro trabajo con el análisis de todos los expedientes de las alumnas que obtuvieron su titulación de enfermeras, el cual algunas lo ampliaron con el de matrona.

Palabras clave: Enfermería, Salus Infirmorum, Tánger.

\section{The Salus Infirmorum School of Nurses at the Spanish Hospital of Tangier}

ABSTRACT: The Salus Infirmorum School of Nurses in Tangier was recognized by the Ministry of Foreign Affairs for the training of women in the field of nursing during the Spanish protectorate. The objective that we set ourselves is to show the overcoming of the regulated training of these students. Our methodology has been based fundamentally on consulting the Salus Infirmorum's own Archives, which have led us to the archives of the Archive of the University of Cádiz, enriching our work with the results of all the records of the students who obtained their degree of nurses and some expanded it with that of midwife.

Keywords: Nursing, Salus Infirmorum, Tangier.

\section{INTRODUCCIÓN}

Este trabajo está fundamentado sobre todo en la consulta de la documentación del Archivo Histórico de Salus Infirmorum de Cádiz (AHSIC) y del Archivo de la Universidad de Cádiz (AUCA), lo que le da veracidad a esta investigación.

La acción de protectorado español sobre una serie de territorios del Sultanato de Marruecos tuvo realmente una duración aproximada de 28 años, ya que entre los años 1912 a 1927 la función de España en la zona fue solo pacificadora.

DOI del artículo: 
Debemos remontarnos atrás en el tiempo y apoyarnos en la consulta de documentos en archivos para entender la labor de la Escuela de Enfermeras de Salus Infirmorum, situada en el Hospital Español de Tánger, entre 1952 y 1959, durante el protectorado español.

En el Protectorado existían otras instituciones sanitarias: Instituto de Higiene de Tetuán, seis hospitales civiles (Tánger, Tetuán, Larache, Alhucemas, Villa Sanjurjo, Nador, Alcazarquivir), un sanatorio marítimo, y un hospital psiquiátrico (Ben Karrich); centros médicos en las ciudades, consultorios médicos y círculos médicos en el campo (Martínez Antonio 2012).

Además, en Tánger, existían diferentes instituciones provenientes de distintos países, entre los que se encontraban: un hospital español (en el que realizaron las prácticas nuestras alumnas), un laboratorio de análisis y dos dispensarios también españoles (Valle Florido y Beni-Makada), dos hospitales franceses (el Instituto Pasteur y el Hospital Benchimol) y uno inglés, uno israelí y otro italiano. Un total, en suma, de nueve instituciones sanitarias para la ciudad de Tánger y zona de alrededor.

Una figura a destacar es la del Padre José $\mathrm{M}^{\mathrm{a}}$ Lerchundi, que nació en Orio (Guipúzcoa) el 24 de febrero de 1836 y murió en Tetuán el 8 de marzo de 1896. Llegó a ser Prior, y en 1859 fue destinado a Marruecos en misiones. A él se le debe la construcción de la iglesia de la calle Siaguin en Tánger, que fue inaugurada en 1881, y la edificación del Hospital Español en el mismo año, que fue remodelado en 1888 y posteriormente refundado en 1952. También creó en Chipiona (Cádiz) un nuevo Colegio en 1882 para proveer de personal a las Misiones de Tierra Santa y Marruecos. Su cuerpo está enterrado en la catedral de Tánger (García Cubillana 2021).

En la Revista de Obras Públicas (noviembre 1942) encontramos referencias a la construcción del citado Hospital, en donde constaba a cargo del ingeniero de caminos José Ochoa y Benjumea al que se le encomendó su construcción. Realizó el estudio del nuevo Hospital Español por encargo del comisario de España en Marruecos Juan Luis Beigbeder y Atienza. Este estudio se desarrolló entre los años 1939 y 1940.

Como Tánger era por aquel entonces ciudad internacional, se contaba con la opinión de muchas naciones para discutir el fin de esta institución y llegaron al acuerdo de utilizarla como una obra benéfico-social.

En los planos del Hospital Español de Tánger podemos apreciar su gran dimensión. Iba a contar con diferentes pabellones, diferenciados según el público al que iban dirigidos: uno para las hermanas que regentarían el edificio, otro para los indígenas y otro pabellón para las personas más pudientes. Estaba dotado con todos los servicios sanitarios que se podían exigir en aquella época, puesto que además de los servicios comunes, como urgencia, hospitalización y consultas, también disponían de un laboratorio propio y un servicio de farmacia. En las consultas, algo a destacar es que, ya que en algunos hospitales carecían de esta comunicación, tenían una puerta de acceso para los enfermos y otra para los médicos, de esta forma podían hacer interconsultas entre ellos.

La ubicación del nuevo Hospital se estableció en el Parque de la Legación de España, que además de ser un lugar bastante amplio, podía atender a nuestros compatriotas dentro del recinto. El lugar más conveniente lo elegían los ingenieros, que eran los que estudiaban el fácil acceso al mismo, que no estuviese desubicado en el tema de la evacuación de aguas residuales, e incluso la orientación de la luz, ya que esto también era beneficioso para los enfermos que estuviesen hospitalizados.

En la actualidad nos podemos encontrar con varios tipos de hospitales, los verticales, que podemos comparar con el Hospital Puerta del Mar de Cádiz; los de pabellones, con el Hospital de Jerez de la Frontera (Cádiz); y los de corredores, como el Hospital de Puerto Real (Cádiz). 
Estos a los que hago mención no son en su totalidad de esta forma, sino que hacen una mezcla, en función de sus necesidades, de los diferentes tipos de hospitales. En el de Tánger se optó por mezclar los de tipo vertical con los de corredores, de esta forma estaban unidas las clínicas por grandes corredores con los servicios generales. Así se conseguían dos cosas, unión entre las clínicas y aislamientos de la hospitalización por los enormes corredores.

También tenemos, no muy lejanos a nosotros, la separación en la hospitalización entre hombres y mujeres. Esto lo podríamos comparar con el antiguo Hospital de Mora de Cádiz, construido en 1903. Sabemos que el Hospital Español de Tánger estaba dividido, según el documento que hemos consultado, en el ala derecha para mujeres y la izquierda para los hombres; los niños también estaban separados de estos últimos. Ambas alas tenían servicios generales independientes, tales como los comedores, sala de espera y recreo, al igual que los cuartos de curas y salas de operaciones. Venía a ser como un pequeño mundo dentro de un gran edificio (figura 1) (Ochoa y Benjumea 1942).

Figura 1. Plano de la planta del Hospital Español de Tánger

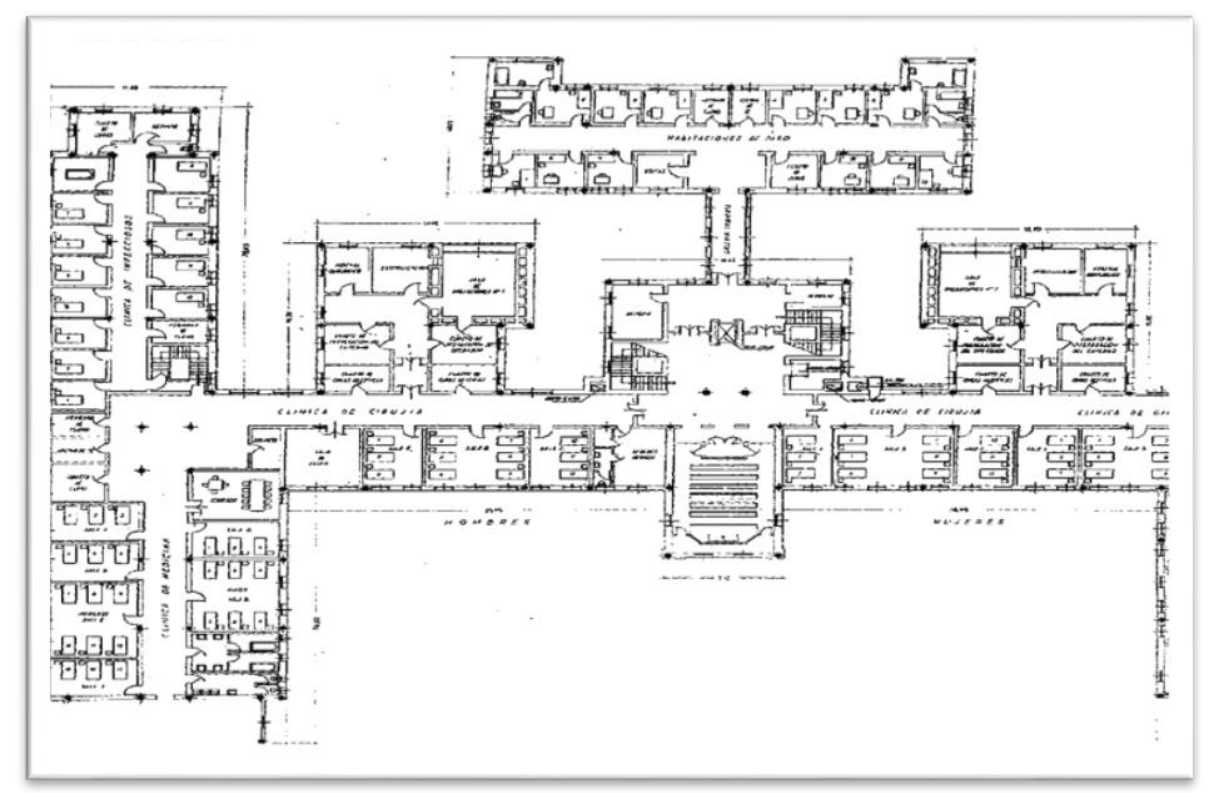

Fuente: OCHOA Y BENJUMEA J., 1942. El nuevo Hospital Español de Tánger. En: Revista de Obras Públicas. Noviembre, pp. 542-548

\section{CREACIÓN DE LA ESCUELA DE ENFERMERAS}

La Hermandad de Enfermeras Salus Infirmorum es una asociación católica que en sus inicios fue precursora en la formación de las mujeres y de futuras profesionales, alcanzando además un reconocimiento y prestigio a través del cuidado a los enfermos en los distintos ámbitos de actuación.

Tras la puesta en marcha de la Hermandad, fue necesaria la creación de centros especializados para poder atender a la formación de estas enfermeras. En Carabanchel (Madrid) en el año 1936 se intentó abrir una casa para poderse reunirse estas enfermeras, pero no fue posible llevarlo a cabo. Es en 1942 cuando se crea la Escuela de Enfermeras, que estuvo vinculada al Hospital del Niño Jesús de Madrid y tuvo como director al Dr. Gregorio Marañón (Conde Mora 2008). Más tarde se crean las de La Coruña, Cádiz, Salamanca, Tánger, Cuenca y Palma de Mallorca. Su labor además de investigadora, era docente y asistencial, sin dejar de la mano la gestión; cuatro palos fundamentales en la que basa hoy en día el rol de la

DOI del artículo:

https://doi.org/10.25267/Cuad investig fondos arch UCA.2021.i3.04 
Enfermería. Dentro de la labor asistencial, estas enfermeras formaban a mujeres sin estudios ni conocimientos sanitarios, con la única intención de que pudieran cuidar a aquel que lo necesitase.

Dentro de las actividades que realizaban las enfermeras estaban las visitas domiciliarias, formando a su vez a familiares para que pudiesen atender al enfermo en los cuidados que requiriese. Atendían suburbios y dispensarios parroquiales donde realizaban labores de curas, inyectables y recomendaciones para prevenir enfermedades. Colaboraban profesionales tituladas de la Facultad de Medicina, que ofrecían, al igual que las enfermeras, su parte humanitaria como voluntarias (Chamorro Rebollo y Martínez de Manuel 2008; Conde Mora 2008).

Esta labor de la Hermandad de Salus Infirmorum se desarrolló en varios sitios de España, extendiéndose al Norte de Marruecos, más exactamente a Tánger, cuando María de Madariaga y Alonso, presidenta de la Escuela de Enfermería Salus Infirmorum, fue llamada desde Tánger por Alberto Martín Artajo, político español miembro de Acción Católica que en 1945 ocupaba el Ministerio de Asuntos Exteriores, y le pidió estudiase la posibilidad de abrir una escuela de Enfermería en el Hospital Español de Tánger para poder formar enfermeras entre las mujeres españolas, marroquíes o francesas, que luego pudieran atender a los españoles que allí residían (figura 2).

Figura 2. Recorte de prensa, marzo 1952

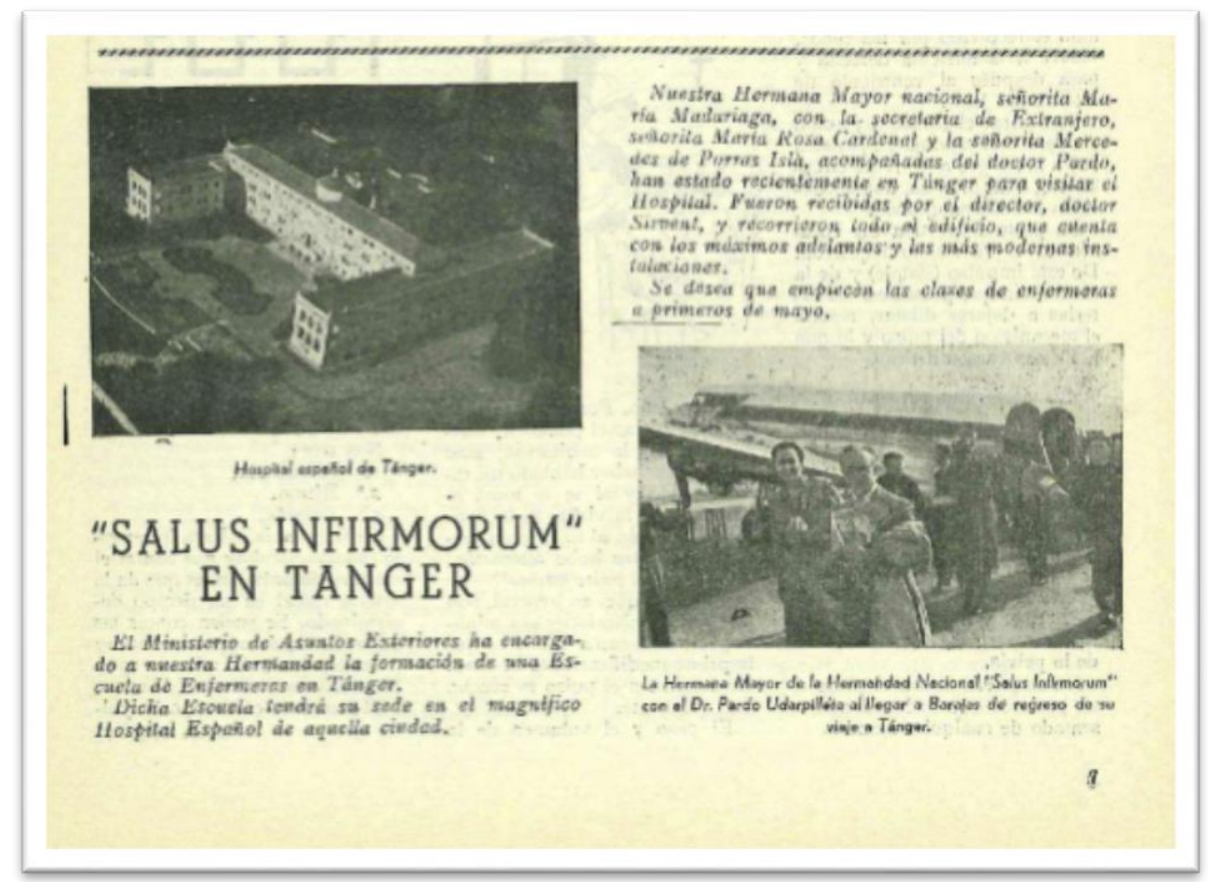

Fuente: Revista Firmes, marzo 1952, tomo I, vol. XX, p. 7

Debemos recordar que durante el Protectorado hubo un auge de traslado de personal sanitario español a Tánger, entre ellos muchas mujeres, que en su gran mayoría eran provisoras de cuidados a los pacientes, encontrándonos gran diferencia con las mujeres marroquíes que seguían un cuidado tradicional en la cura de los enfermos; es por ello la emergencia de la formación de las mujeres marroquíes para atender el aumento de personas que había alcanzado el país (Jiménez-Lucena 2006).

El 10 de enero de 1952 Teodoro Ruiz de Cuevas, secretario de la Embajada de España en Marruecos, por mandato del Ministerio de Asuntos Exteriores, permite la creación de la 
Escuela de Enfermeras Españolas de Salus Infirmorum en el Hospital Español de Tánger, a cargo de la Hermandad de Salus Infirmorum presidida por María de Madariaga y Alonso.

\section{LAS ALUMNAS DE ENFERMERÍA DE SALUS INFIRMORUM}

Una vez recibida la autorización, María de Madariaga y Alonso comienza con todos los trámites, y tras realizar una campaña publicitaria en periódicos de Tánger, en 1952, para conocer el nivel de formación que tenían las mujeres aspirantes, convoca un curso preliminar con una duración de 10 semanas, donde además de la formación teórica recibían una formación de prácticas clínicas de 4 horas diarias.

María de Madariaga, una vez finalizado el curso preliminar, y viendo el buen resultado obtenido, inaugura la Escuela de Enfermería en el Hospital Español de Tánger el año 1953 con 20 alumnas de distintas nacionalidades.

Para entrar en la Escuela de Enfermería de Salus Infirmorum en Tánger todas las alumnas tenían que superar una prueba que se realizaba en la misma Escuela tras la finalización de estos cursillos preliminares, para verificar que habían adquirido los conocimientos necesarios.

En el Archivo de Salus Infirmorum de Cádiz se conserva la documentación referente a este curso preliminar. Las materias que debían cursarse eran: Religión, Elemento Moral Profesional, Anatomía y Fisiología, Técnicas y Cuidado de Enfermos, Formación Política y Hogar. Además de la parte teórica, tenían formación práctica con 4 horas diarias en la sala de demostraciones y en salas de hospitalización.

La duración del curso preliminar era de 10 semanas, correspondientes a 60 días, y organizado semanalmente: formación teórica 2 horas diarias, 5 veces a la semana, con un total de 10 horas semanales; formación practica 4 horas diarias con un total de 20 horas semanales.

El curso preliminar constaba de un total de 340 horas, de las que 100 corresponderían a materia teórica y 240 a prácticas clínicas (tabla I).

Tabla I. Materias prácticas (4 horas diarias)

\begin{tabular}{llr} 
& LUGAR & SEMANAS \\
\cline { 2 - 3 } PráCTICAS & Sala de demostraciones & 4 \\
\cline { 2 - 3 } (4 horas diarias) & Medicina General & 4 \\
\cline { 2 - 3 } & Pediatría & 1 \\
\cline { 2 - 3 } & Cirugía & 1
\end{tabular}

Fuente: Elaboración propia basada en AHSIC, Sección Tánger (1952-1959)

En el 1. ${ }^{\text {er }}$ curso (1952-1953) de Salus Infirmorum en Tánger se matricularon 25 alumnas, según el análisis de la documentación del archivo de Salus Infirmorum de Cádiz. Las alumnas que finalizaron ese primer curso de enfermería fueron un total de 20 (tabla II). Se desconocen los motivos por los cuales 5 alumnas abandonaron la carrera. 
Tabla II. Alumnas del 1. ${ }^{\mathrm{er}}$ curso de Enfermería (1952-1953)

\begin{tabular}{l|l} 
ALUMNAS DE $1 .{ }^{\text {er }}$ AÑO BECADAS & ALUMNAS DE $1{ }^{\text {er }}$ AÑO SIN BECAR \\
\hline Ana Montero Godinez & $\mathrm{M}^{\mathrm{a}}$ Luisa Lajusticia \\
\hline María Dolores Flecha Ruiz & $\mathrm{M}^{\mathrm{a}}$ Dolores Medrano \\
\hline Clara Pinto de Toledano & Ana $\mathrm{M}^{\mathrm{a}}$ Miller \\
\hline Sohora Adselam & Edith Márquez \\
\hline Fatima Dailali & Ángeles Erro \\
\hline Amina Dailali & $\mathrm{M}^{\mathrm{a}}$ Gracia Mancheciano \\
\hline Fatima Sihiri & Carmen Díaz \\
\hline Leonor Maria Homen de Mello & Lyna Azagury \\
\hline Josefa Martínez & Marta García \\
\hline & Lylyan Esayag \\
\hline & Anita Lasry
\end{tabular}

Fuente: Elaboración propia basada en AHSIC, Sección Tánger (1952-1959)

Además del examen de final de curso, las pruebas durante la formación eran de carácter trimestral y de naturaleza teórico-práctica (figuras 3 y 4) y las calificaciones finales se entregaban en un acto privado en el Hospital Español de Tánger. Estos exámenes eran realizados por el mismo profesorado que impartía las asignaturas.

Figuras 3 y 4. Ejemplos de material teórico para la aplicación en las prácticas clínicas
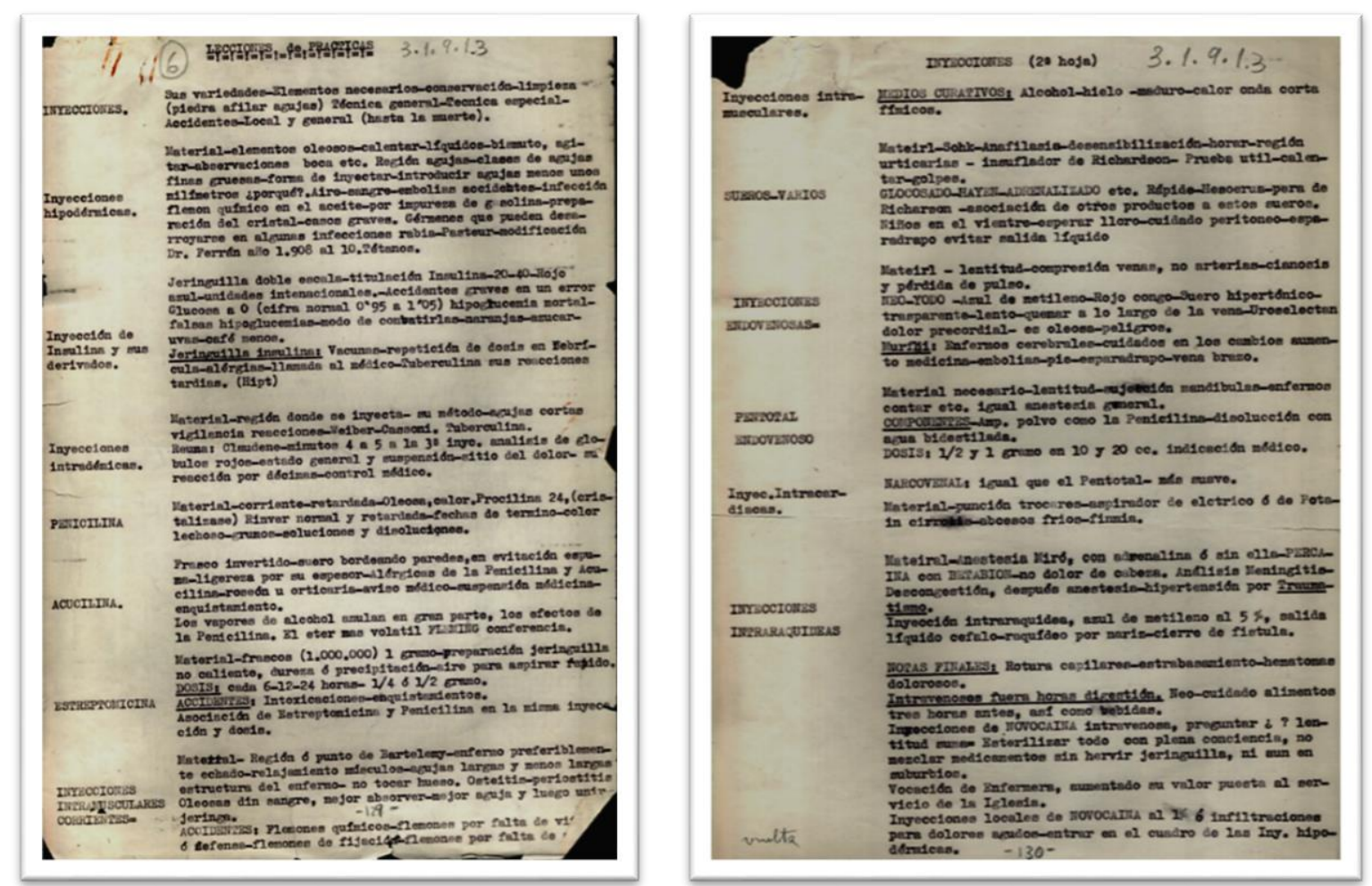

Fuente: AHSIC, Sección Tánger, 3.1.9.1.3. Tema de Prácticas de Inyecciones 1 y 3.1.9.1.3. Tema de Prácticas de Inyecciones 2

DOI del artículo: 
Algunas de las alumnas tuvieron que superar la barrera del idioma y, por ello, dedicar parte de su tiempo de formación para aprender el español, tanto escrito como hablado. Podemos ver en el apartado de apéndice documental, en algunas de las cartas de las alumnas enviadas desde Tánger a la Hermana Mayor, su agradecimiento por la implicación de las hermanas en ese aprendizaje. Incluso algunas de ellas tuvieron que aprender a sumar y restar o escribir en una gráfica.

Al tratarse de estudios no reglados, las alumnas, tras la finalización de cada curso, debían desplazarse a la Facultad de Medicina de Cádiz para la realización del examen oficial, debiendo aportar también diversa documentación: certificado de nacimiento, certificado de examen médico en el que figurase la ausencia de enfermedades contagiosas, además de estar correctamente vacunada y documento acreditando que la alumna había realizado las prácticas clínicas correspondiente al curso académico.

Hay que indicar que, inicialmente, los estudios de Enfermería en Tánger constaban de dos años y no fue hasta el año 1953 cuando la carrera de Enfermería pasa a ser de 3 cursos (tablas III, IV y V).

Primer curso: La duración del curso era de 9 meses con un total de 105 días. Las prácticas consistían en 4 horas diarias en las salas de enfermos. El programa de estudios para este primer curso incluía las asignaturas de Religión, Ética Profesional, Moral, Nociones de Anatomía y Fisiología, Higiene, Física y Química, Cultura General y Cocina Dietética.

Tabla III. Asignaturas del primer curso. Clases teóricas

\begin{tabular}{lr} 
ASIGNATURAS & HORAS TOTALES \\
\hline Religión & 21 \\
\hline Moral Profesional & 21 \\
\hline Hogar & 21 \\
\hline Formación Política & 21 \\
\hline Anatomía y Fisiología & 42 \\
\hline Técnica de Cuidado de Enfermos & 21 \\
\hline Bacteriología & 12 \\
\hline Higiene & 15 \\
\hline Elementos Física y Química & 15 \\
\hline Matemáticas Aplicadas & 15 \\
\hline Historia de la Profesión & 6
\end{tabular}

Fuente: Elaboración propia basada en AHSIC, Sección Tánger (1952-1959)

Segundo y tercer curso: ambos cursos muy equiparados, tanto en horas como en duración, ocupaban un total de 165 días. Las prácticas aumentaban con respecto al curso de primero. Las alumnas debían estar 6 horas diarias en la sala de los enfermos con un total de 30 horas semanales. 
Tabla IV. Asignaturas del segundo curso. Clases teóricas

ASIGNATURAS

HORAS TOTALES

\begin{tabular}{lr}
\hline Religión & 31 \\
\hline Moral Profesional & 31 \\
\hline Hogar & 31 \\
\hline Formación Política & 31 \\
\hline Patología & 73 \\
\hline Farmacología & 15 \\
\hline Dietética & 31 \\
\hline Química & 31 \\
\hline Psicología & 31 \\
\hline Historia de la Profesión & 6
\end{tabular}

Fuente: Elaboración propia basada en AHSIC, Sección Tánger (1952-1959)

Tabla V. Asignaturas del tercer curso. Clases teóricas

ASIGNATURAS

HORAS

TOTALES

\begin{tabular}{ll}
\hline Religión & 31 \\
\hline Moral Profesional & 31 \\
\hline Hogar & 31 \\
\hline Formación Política & 31 \\
\hline Enfermedades Infecciosas & 31 \\
\hline Órganos Sentidos & 31 \\
\hline Fisioterapia & 15 \\
\hline Ginecología y Puericultura & 31 \\
\hline Higiene y Psiquiatría & 31 \\
\hline Psicología & 31 \\
\hline Sociología & 31 \\
\hline Orientación Sanitaria & 15
\end{tabular}

Fuente: Elaboración propia basada en AHSIC, Sección Tánger (1952-1959)

La primera promoción de enfermeras (1952-1954) tuvo así que ampliar su formación en un año más para poder validar la carrera de Enfermería. La mayoría de las alumnas realizaron ese tercer curso en la propia ciudad de Tánger, y tras su finalización se desplazaban a la ciudad de Cádiz para realizar el examen en la Facultad de Medicina y poder obtener el Título Oficial de Enfermería.

Transcurrido el primer curso (1952-1953) de Enfermería, el personal directivo del Hospital Español elogió las técnicas y moral de las alumnas, así como el riguroso 
cumplimiento por parte de la Escuela de las normas correspondientes al Decreto de 1952 sobre Escuelas de Enfermería, remitiendo al Ministerio de Educación la documentación necesaria para el Registro Oficial de la Escuela de Enfermeras Salus Infirmorum en el Hospital Español de Tánger.

Tras dos años de formación, en 1953, egresó la primera promoción de la Escuela, promoción que contó con 20 nuevas enfermeras.

Cabe señalar que las calificaciones finales obtenidas tras el período formativo no permitían a las egresadas ejercer como enfermeras, ya que se trataba, como comentamos anteriormente, de una formación no reglada. Para obtener el Título Oficial, las alumnas debían presentarse, como ya explicamos, a examen oficial en la Facultad de Medicina de Cádiz y disponer así del título de Ayudante Técnico de Enfermería.

Los expedientes de esas alumnas se encuentran en el Fondo de la Facultad de Medicina del Archivo de la Universidad de Cádiz. Según hemos podido comprobar en el citado Fondo, en el curso 1952/53, 20 alumnas se trasladaron desde Tánger a Cádiz para realizar sus exámenes finales de primer año, acompañadas de la Presidenta Nacional y de la Jefa Auxiliar de la Escuela. Estas 20 estudiantes, entre las que se encontraban marroquíes, italianas, portuguesas y españolas, aprobaron en su totalidad su primer curso, obteniendo 4 de ellas la calificación de sobresaliente, 4 la de notable y el resto la de aprobado.

A lo largo de los cursos 1952-1957 pasaron por la Escuela de Enfermeras 3 promociones, con un total de 42 alumnas de las que finalizaron los estudios 36 de ellas (tabla VI).

Tabla VI. $N^{o}$ de alumnas que ingresan y egresadas, 1952-1957

PROMOCIÓN N N. DE ALUMNAS AL INGRESO N $\quad{ }^{\circ}$ DE ALUMNAS EGRESADAS

\begin{tabular}{lrr}
\hline $1952-1954$ & 25 & 20 \\
\hline $1953-1956$ & 8 & 7 \\
\hline $1954-1957$ & 9 & 9 \\
\hline TOTAL & 42 & 36
\end{tabular}

Fuente: Elaboración propia basada en AHSIC, Sección Tánger (1952-1959)

La documentación que se conserva en el Archivo Histórico de la Universidad de Cádiz acredita la formación sanitaria de alumnas en el Hospital de Ifni, en concreto, tenemos el caso de Fátima Ben Ahmed Ben Mehand que durante el curso 1947-1949 realizó el examen en la Facultad de Medicina de Cádiz para el Titulo de Practicante, propio a los cursos de $1^{\circ}$ y $2^{\circ}$. Debemos resaltar que dicha alumna quedó exenta del servicio social, obligatorio para el resto de las alumnas españolas, atendiendo al régimen especial del África Occidental española (figura 5).

La superación del Bachillerato español daba acceso a las universidades y escuelas superiores en España. Además, en Tetuán, tras tres cursos de bachillerato, podían seguir los estudios en la Escuela Politécnica española en la que se desarrollaban estudios de corta duración (peritaje agrícola, peritaje comercial, estudios administrativos, de auxiliares de Medicina, practicantes o comadronas). Los alumnos aventajados de la Escuela Politécnica tenían así la posibilidad de ejercer funciones en la Administración del Protectorado (Vilar 2008). 
Figura 5. Telegrama comunicando exención de servicio social Fátima Ben Ahmed Ben Mehand (julio 1949)

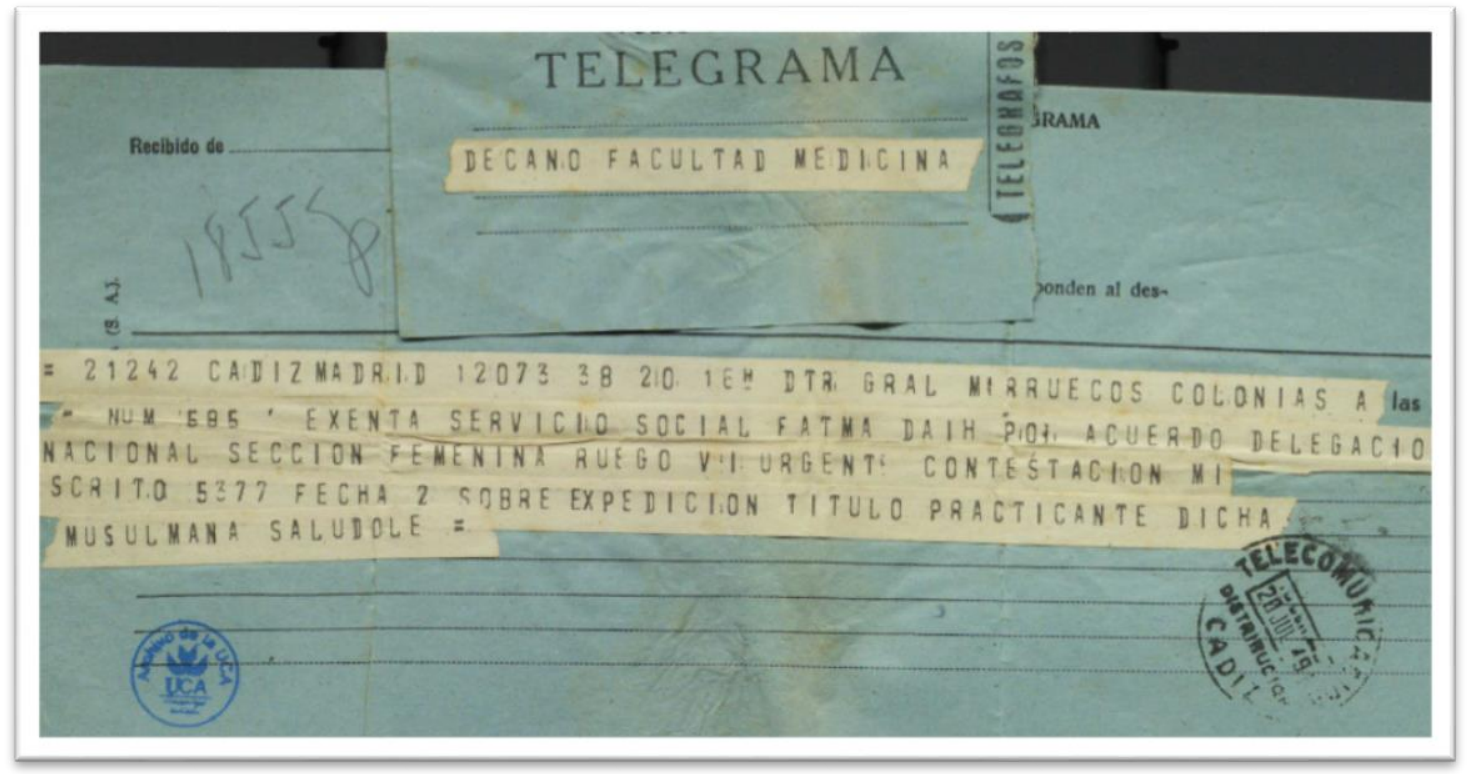

Fuente: Archivo de la Universidad de Cádiz (AUCA). Expediente personal de Fátima Ben Ahmed Ben Mehand, Practicante, 1948-1949, C-407 (3)-52 FM

\section{APÉNDICE DOCUMENTAL}

En este pequeño apéndice documental, quiero poner de relieve las fuentes de esta investigación, donde podemos ver en imágenes y documentos una pequeña muestra de las alumnas que alcanzaron su objetivo, a pesar de las adversidades del idioma, la cultura y la religión, donde pongo de manifiesto la labor de Salus Infirmorum en su Escuela de Enfermeras en el Norte de Marruecos, de donde salieron unas excelentes enfermeras, tanto a nivel humano como profesional, alcanzando su meta para poder atender a aquellas personas que necesitasen de sus cuidados. «El camino nunca es fácil, pero con cariño e ilusión siempre se consigue». 
Figura 6. Grupo de Alumnas de Tánger con Maria de Madariaga y Alonso (fundadora de la Escuela de Salus Infirmorum) en el centro. Tánger, 1952

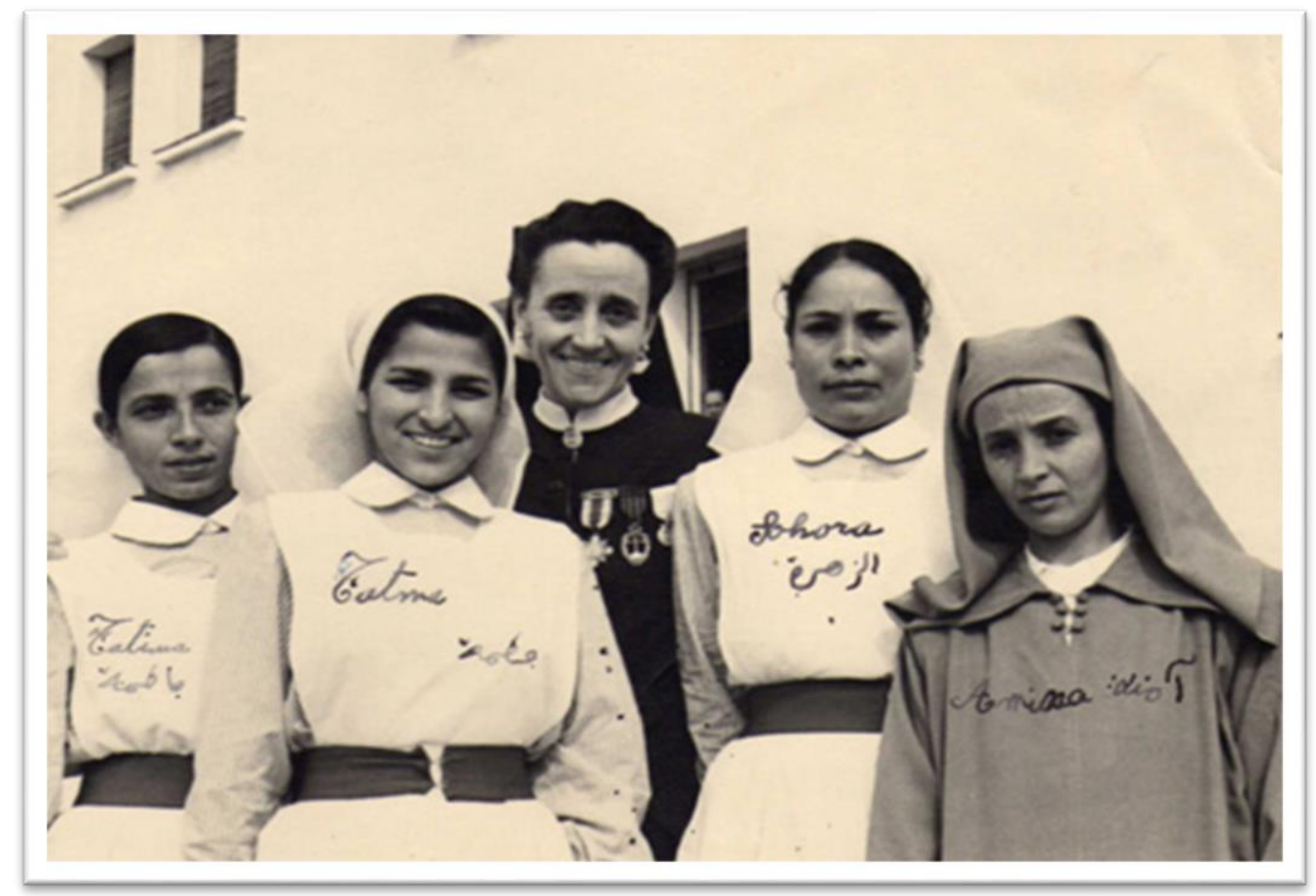

Fuente: Archivo Histórico Salus Infirmorum de Cádiz (AHSIC)

Figuras 7 y 8. Pago de Matricula y de Titulo de una de las alumnas de las foto. Tánger, 10 junio 1953 y 16 de enero de 1956

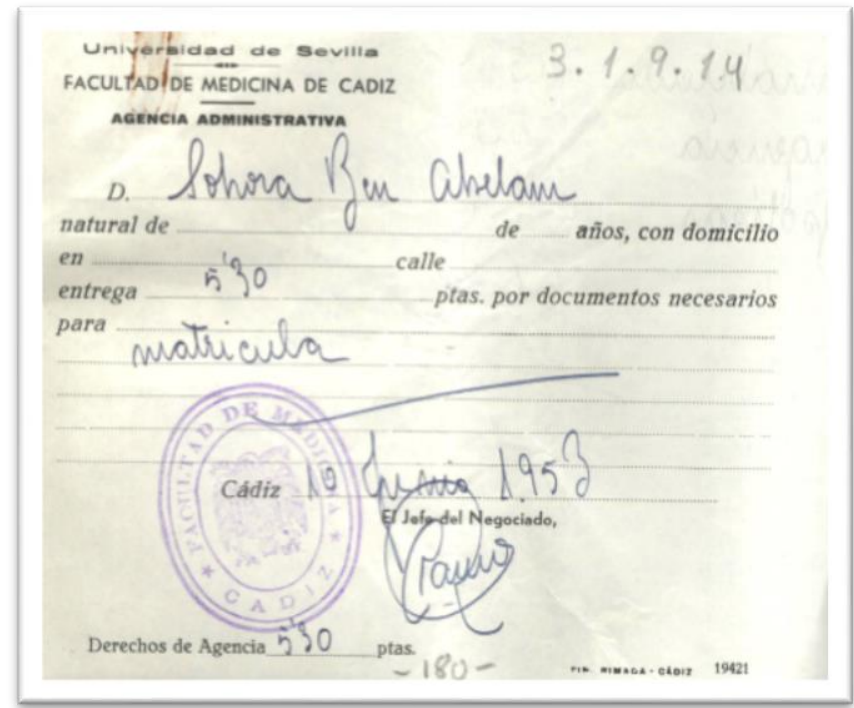




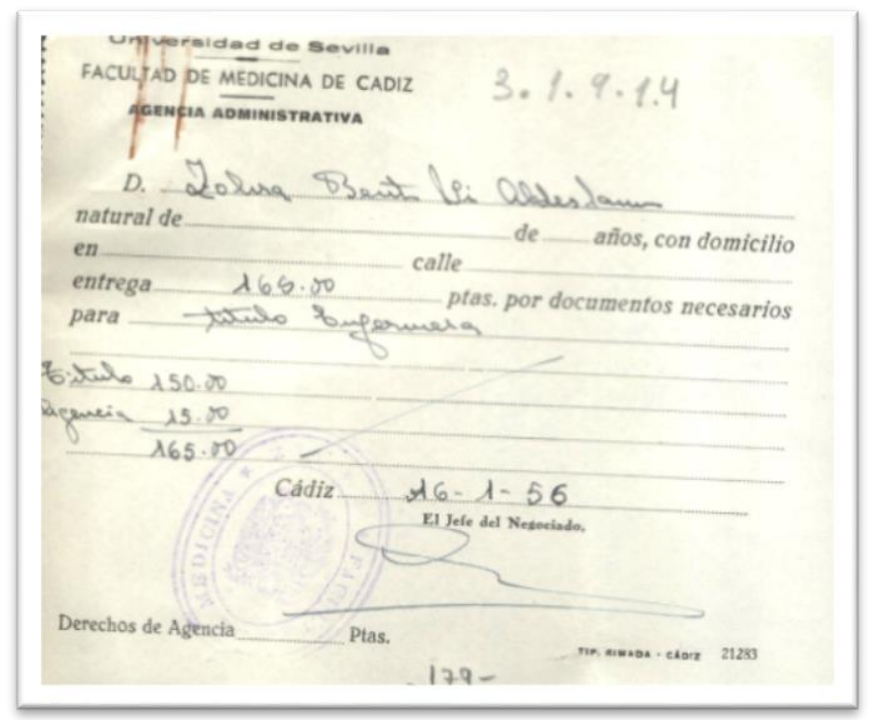

Fuente: Archivo Histórico Salus Infirmorum de Cádiz (AHSIC)

Figuras 9 y 10. Escrito de una de las alumnas dirigido a la Hermana Mayor (María de Madariaga) donde le demuestra su agradecimiento por lo aprendido, Tánger, 27 agosto de 1953.
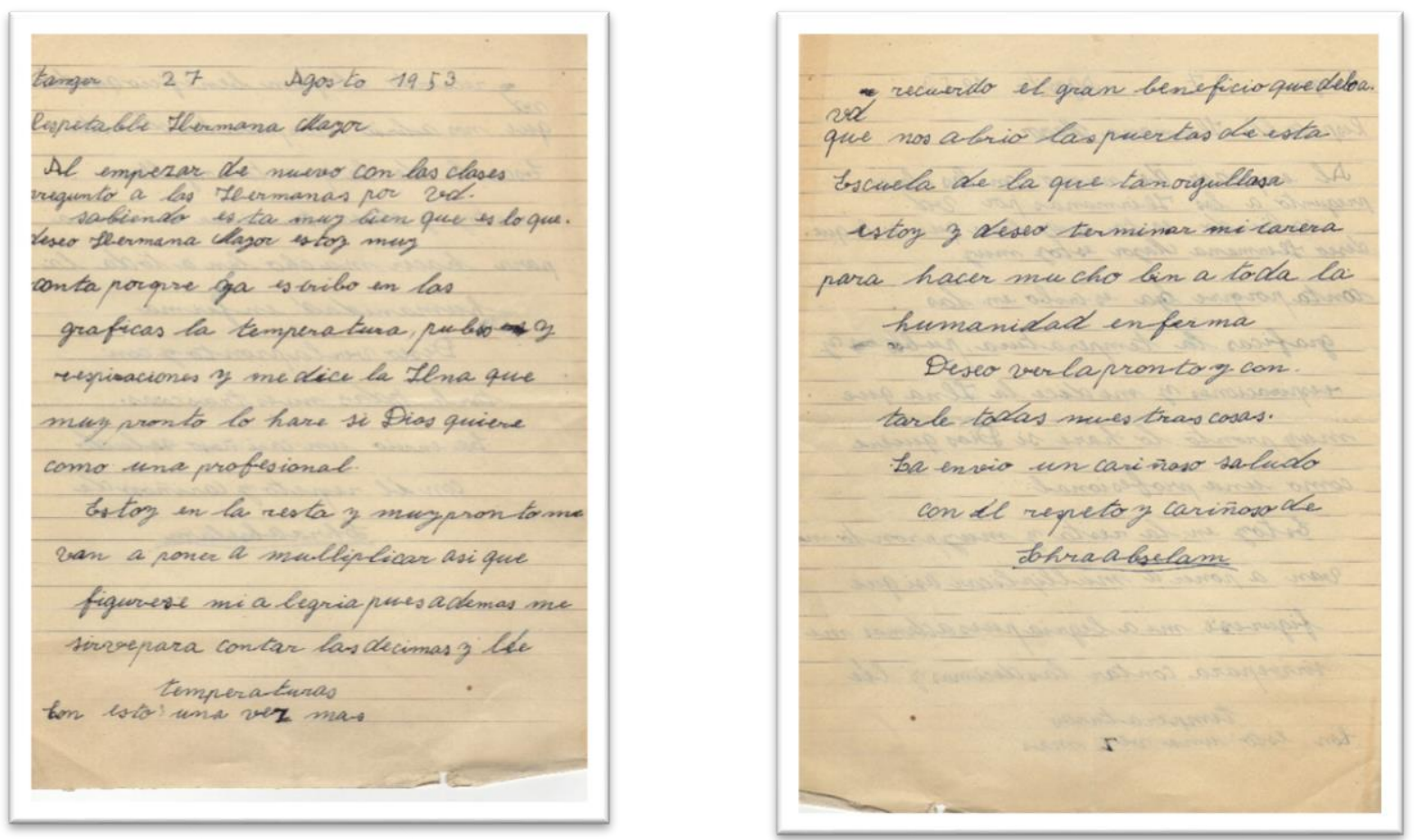

Fuente: Archivo Histórico Salus Infirmorum de Cádiz (AHSIC) 
Figuras 11 y 12. Certificado expedido por Carlos Sirvent, director del Hospital y de la Escuela de Tánger, a favor de la alumna Amina Ben Abselam (alumna fotografiada, figura 6) de que ha efectuado su examen de ingreso para darle acceso a seguir sus estudios; expediente de la alumna que, tras su esfuerzo, consigue el título de enfermera en mayo de 1961.
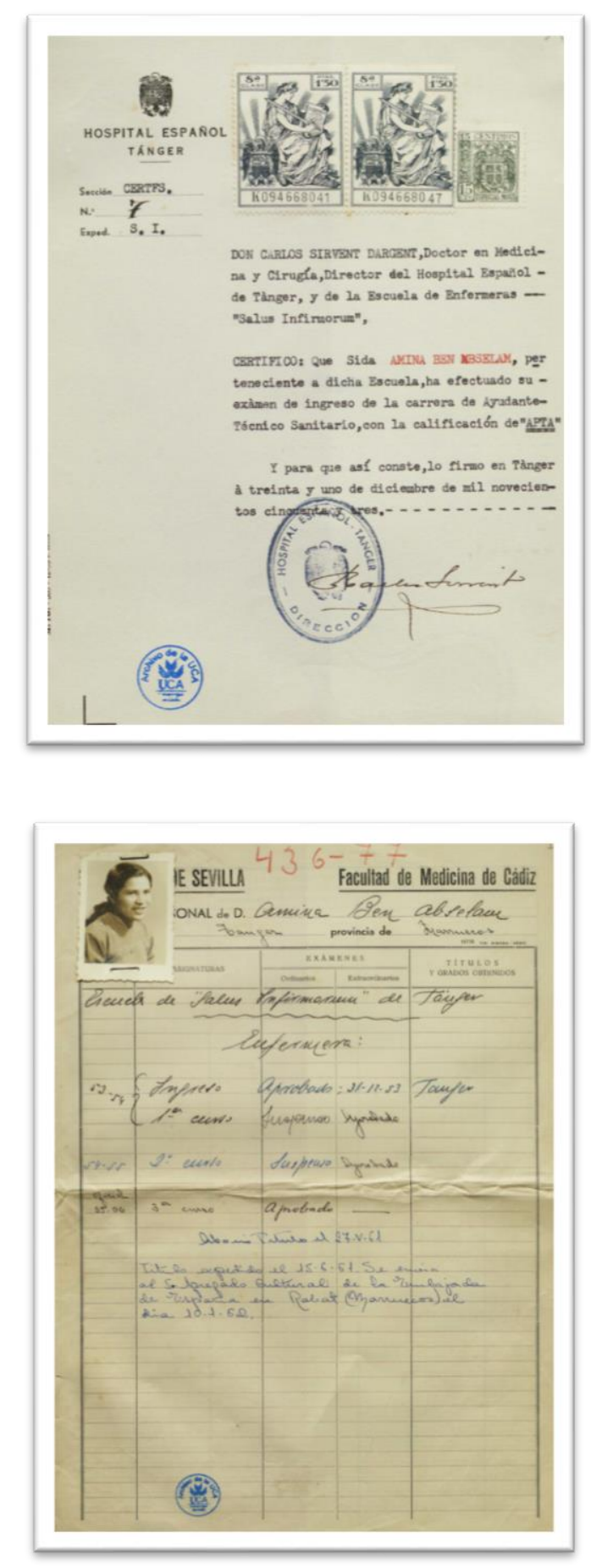

Fuente: Archivo de la Universidad de Cádiz (AUCA). Expediente personal de Amina Ben Abselam [Abdslan] Lachiri, 1953-1961, C-436 (3)-77 FM 
Figuras 13 y 14. Expediente de las dos hermanas que veíamos en el primer documento fotográfico (figura 6), donde alcanzan su titulación por parte de la Facultad de Medicina de Cádiz, el aprobado de Cuidadoras Profesionales, julio 1955 y julio de 1954.
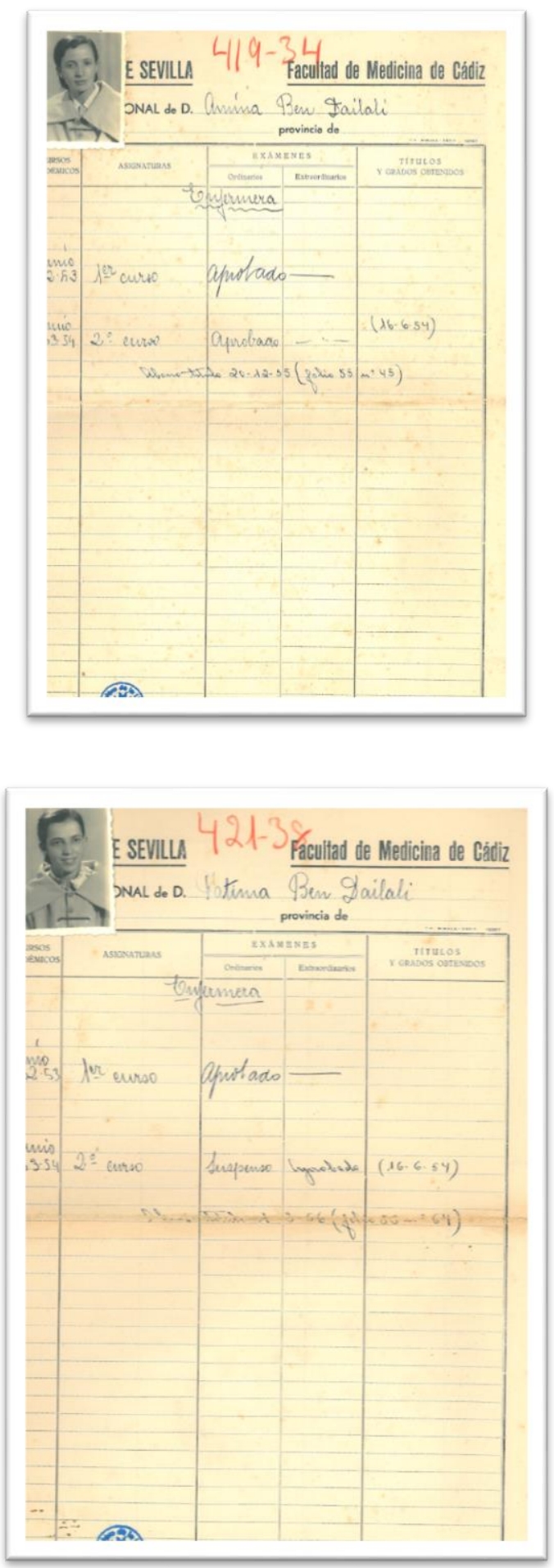

Fuente: Archivo Universidad de Cádiz (AUCA). Expediente personal de Amina Ben Dailali, 1952-1954, C-419 (2)-34 FM; Expediente personal de Fátima Ben Dailali, 1952-1954, C-421 (2)-38 FM 
Figura 15. Programa de la carrera de Ayudante Técnico Sanitaria (Enfermeras), incluyendo el curso preliminar.

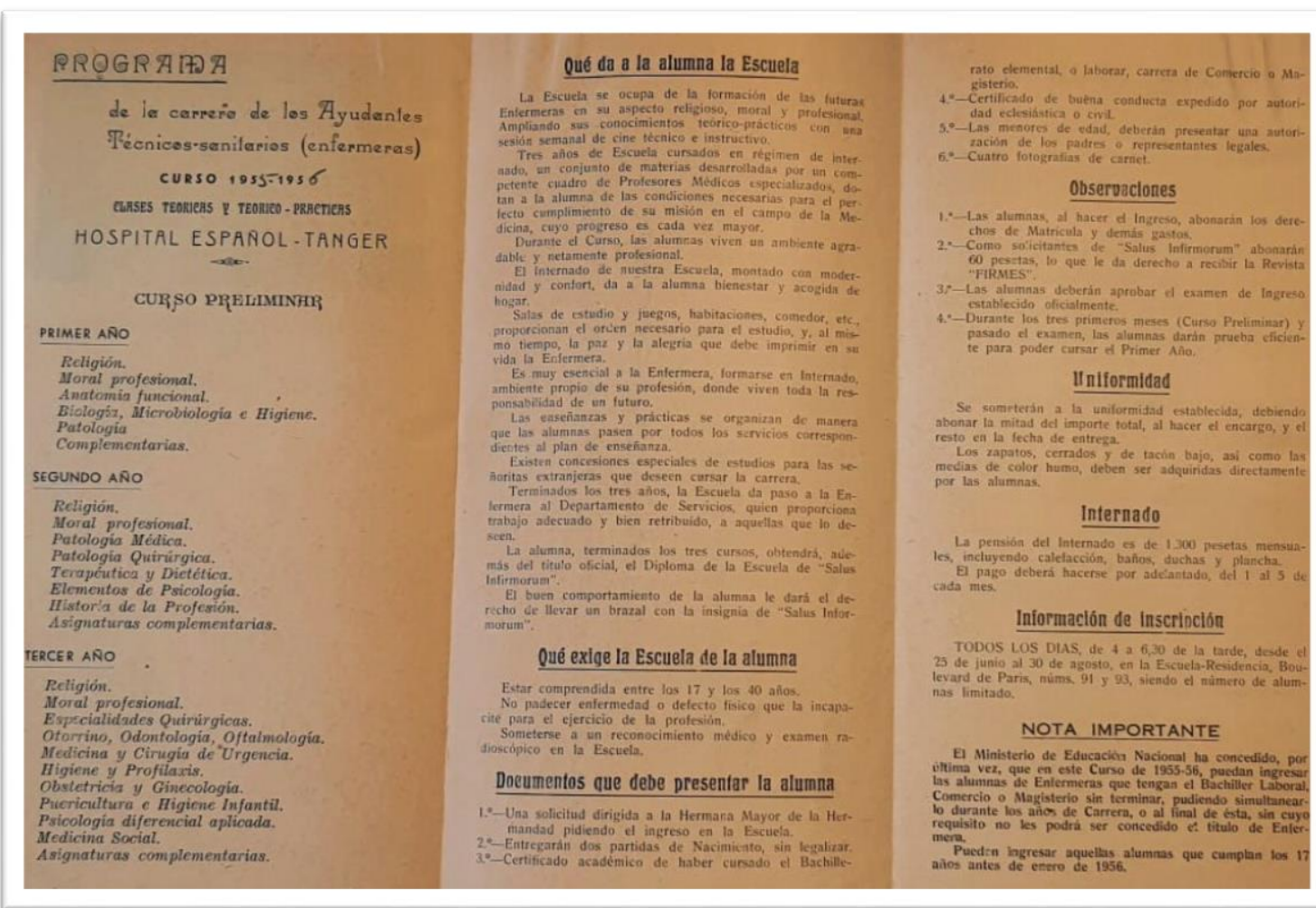

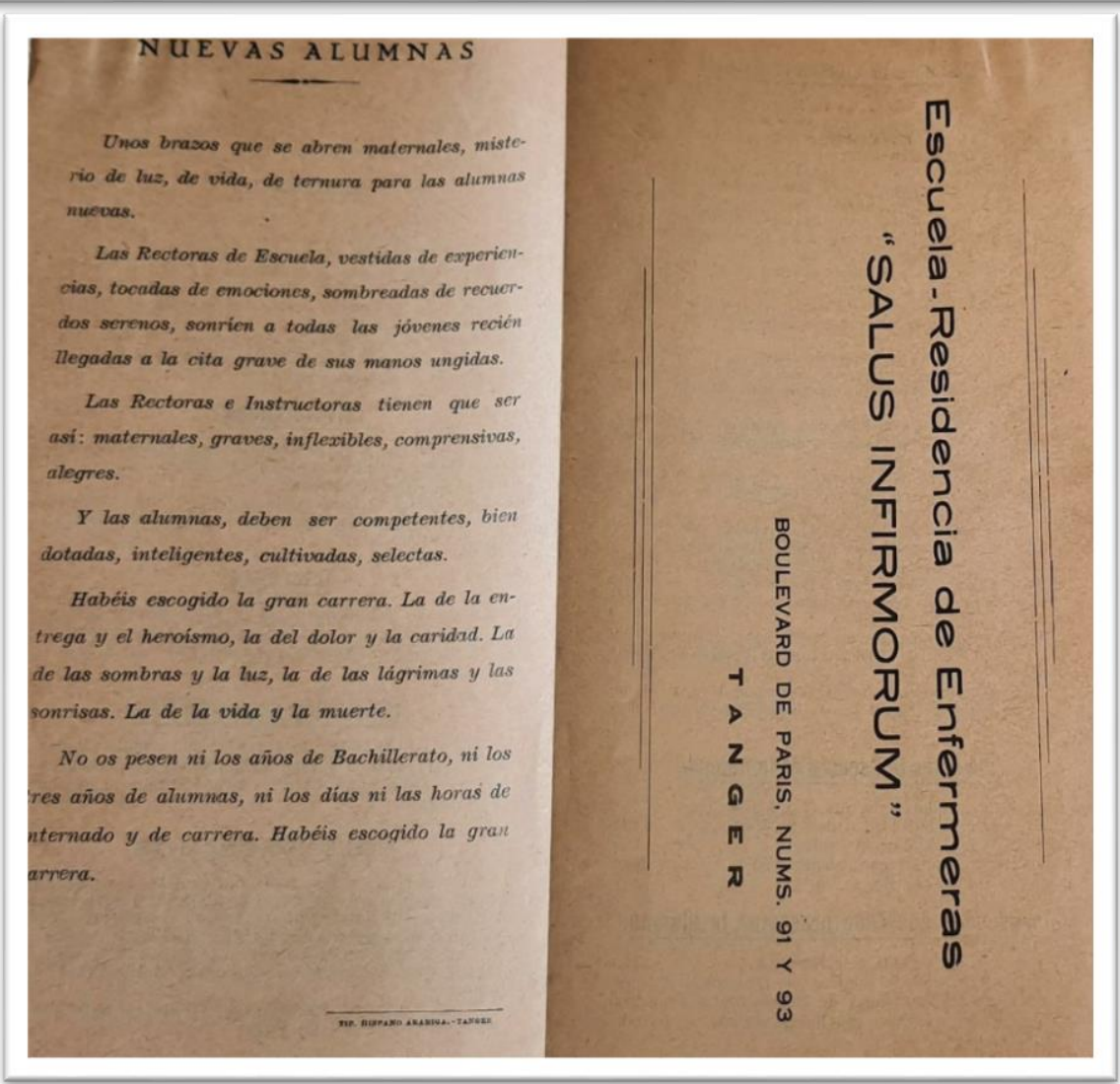

Fuente: Archivo Histórico Salus Infirmorum de Cádiz (AHSIC), Sección Tánger (1952-1959)

DOI del artículo:

https://doi.org/10.25267/Cuad investig fondos arch UCA.2021.i3.04
Editorial [L] UCA REVISTAS | Universidad de Cádiz 


\section{REFERENCIAS BIBLIOGRÁFICAS}

Conde Mora, F. G., 2008. La Hermana Mayor Da María de Madariaga Alonso (1905-2001). Cádiz, Salus Infirmorum.

Chamorro Rebollo, E. y Martínez de Manuel, A., 2008. Del ayer al hoy, de María de Madariaga a "Salus Infirmorum". En Híades: Revista de historia de la enfermería, n. 10, 2. (Ejemplar dedicado a: Tradición y Modernidad. Las fuentes para el estudio de la Historia de la Enfermería), pp. 1151-1163.

Garcia Cubillana, J. M., 2021. La Sanidad Militar Española en el Norte de África y sus relaciones con la Orden Franciscana Menor (1877-1926). En: Revista Ars Clínica. Académica de la Real Academia de Canarias, vol. 6, n. ${ }^{\circ}$ 2, pp.5-19

JimÉnEZ-LuCENA, I., 2006. Género, Sanidad y Colonialidad: La mujer marroquí y la mujer española en la política sanitaria de España en Marruecos. En: História, Ciências, Saúde-Manguinhos, vol. 13 (2): 325-47.

MARTíneZ ANTONIO, F. J., 2012. La medicina y la sanidad en el protectorado español en Marruecos. Apuntes iconográficos. En: Estudios sobre el mundo árabe e islámico contemporáneo, $\mathrm{n}^{\circ}$ 5-6, pp.135-156. ISSN 0214-834X.

Mata Perez, C., LePiani Díaz, I. y Calatayud Succino, J., 2015. Salus Infirmorum en Tánger en los años 1952-1956. En: XIV Congreso Nacional y IX Internacional de Historia de Enfermería "Un siglo cuidando a la Sociedad Centenario del reconocimiento oficial de la Enfermería en España". Colegio de Enfermería de Cantabria, pp. 297-300. ISBN: 978-84-606-7630-0.

Miralles SANGRO, Ma ${ }^{\text {a }}$ T. et al. Historia de la Escuela de Enfermeras Salus Infirmorum en Madrid". En: Cultura de los Cuidados. Revista de Enfermería y Humanidades, $\mathrm{n}^{\circ} 1$ y 2. Seminario de Historia y Antropología de los Cuidados Enfermeros. Alicante: Universidad de Alicante, 1997.

OchoA y Benjumea J., 1942. El nuevo Hospital Español de Tánger. En: Revista de obras públicas. Noviembre, pp. 542-548.

ORTIZ GómEZ, T., 2004. Interacciones entre salud, historia y feminismo. Una revisión historiográfica. Reflexión Y Crítica. En: Diálogo Filosófico, n. o 59, pp. 229-244.

VILAR, J. B., 2008. Las fuentes españolas, francesas y magrebíes para el estudio de las relaciones internacionales contemporáneas de España con Marruecos, Argelia, Túnez y Libia: Una aproximación. En: Espacio, Tiempo y Forma, Serie V, Historia Contemporánea, Ed. UNED, t. 20. pp. 185-206. 